\title{
Reorientation by features and geometry: Effects of healthy and degenerative age-related cognitive decline
}

\author{
Kevin Leonard ${ }^{1} \cdot$ Viktoriya Vasylkiv $^{1} \cdot$ Debbie M. Kelly $^{1,2}$ \\ Published online: 8 January 2020 \\ (C) The Psychonomic Society, Inc. 2020
}

\begin{abstract}
The ability to orient is critical for mobile species. Two visual cues, geometry (e.g., distance and direction) and features (e.g., colour and texture) are often used when establishing one's orientation. Previous research has shown the use of these cues, in particular, geometry, may decline with healthy aging. Few studies have examined whether degenerative aging processes show similar time points for the decline of geometry use. The present study examined this issue by training adult and aged mice from two strains, a healthy wild-type and an Alzheimer's model, to search for a hidden platform in a rectangular water maze. The shape of the maze provided geometric information, and distinctive features were displayed on the walls. Following training, manipulations to the features were made to examine whether the mice were able to use the features and geometry, and whether they showed a preference between these two cue types. Results showed that although Alzheimer's transgenic mice were slower to learn the task, overall age rather than strain, was associated with a degradation in use of geometry. However, the presence of seemingly uninformative features (due to their redundancy) facilitated the use of geometry. Additionally, when features and geometry provided conflicting information, only young wild-type mice showed a primary use of features. Our results suggest the failure to use geometry may be a generalized function of aging, and not a diagnostic feature of degeneration for mice. Whether this is also the case for other mammals, such as humans for which the mouse is an important medical model, remains to be examined.
\end{abstract}

Keywords Comparative cognition $\cdot$ Configural learning $\cdot$ Cue competition $\cdot$ Spatial learning $\cdot$ Reorientation

A critical component of survival for most mobile animals is the ability to orient (Cheng \& Newcombe, 2005) - that is, to determine one's position relative to objects and locations within an environment. Indeed, establishing one's position is the necessary first step for navigation. One key aspect of orientation in humans, and many other species, is the use of visual cues. Two of the most commonly studied visual cues for understanding spatial orientation are features and geometry (e.g., Cheng, 1986; Kelly, Spetch, \& Heth, 1998; Vallortigara, Zanforlin, \& Pasti, 1990). Featural cues include the properties of objects or surfaces, such as colours or patterns, whereas geometric cues include the metric configurations and relationships among objects or surfaces (e.g., distance and direction).

Debbie M. Kelly

Debbie.Kelly@umanitoba.ca

1 Department of Psychology, University of Manitoba, 190 Dysart Road, Winnipeg, MB R3T 2N2, Canada

2 Department of Biological Sciences, University of Manitoba, Winnipeg, Manitoba, Canada
The importance of these two types of visual cues was first identified by Cheng (1986), during a study on the reorientation ability of rats. In particular, during the working-memory task, rats were trained to search for a hidden food reward in one corner of a rectangular environment that contained discrete and distinct features (panels with unique colour, texture, and odor), one placed in each of the four corners. Cheng observed that the rats would make systematic errors, whereby they would search at the trained corner as well as at the diagonally opposite corner in equal proportions. Although the diagonally opposite corner contained a distinctively different featural panel, it shared the same geometric properties as the trained corner (a long wall to the right side, and a short wall to the left side, for instance). Hence, Cheng termed these systematic errors rotational errors. The distinctive featural cues in each corner allowed for potentially perfect task accuracy if the rats only attended to the featural cues. Yet they showed an incidental encoding of environmental geometry alongside learning the features. Subsequent experiments using similar paradigms - namely, reorientation in a rectangular environment containing distinct featural 
cues-have shown a similar incidental encoding of geometry across a wide variety of species (e.g., Cheng, 1986; Kelly et al., 1998; Vallortigara et al., 1990; for a review, see Cheng, Huttenlocher, \& Newcombe, 2013; Cheng \& Newcombe, 2005).

The domestic mouse (Mus musculus) has recently become the subject of increased interest for the study of spatial orientation for reasons such as the potential to create knock-down and knock-out mouse models of human disorders, and their relatively short life span, which together has allowed for a better understanding of the neurological mechanisms supporting healthy and degenerative changes in cognition and behaviour across the life span (O'Connor, Huber, \& Svoboda, 2009; Price, Sisodia, \& Borchelt, 1998). For instance, the development of mouse models for human disorders with associated spatial orientation deficits, such as PraderWilli syndrome (Lee et al., 2015) and Alzheimer's disease, has informed our understanding of the neurological changes associated with spatial degeneration (Bilkei-Gorzo, 2014; Hardy \& Selkoe, 2002). Establishing a foundational understanding of the reorientation abilities of neurotypical mice, and how these reorientation abilities might change across the life span, is a necessary precursor to studies using generic mouse models of spatial degeneration. Previous research has shown that mice, similar to other species studied to date, encode features and geometry when reorienting (Leonard, Tian, Ivanco, \& Kelly, 2018; Twyman, Newcombe, \& Gould, 2009), and that there are nuances to the ways in which mice use the two cue types. Mice will more readily use featural cues that differ along a scalar dimension (e.g., size of pattern elements) to orient, rather than featural cues that differ categorically (e.g., colour; Twyman et al., 2009). The encoding of scalar information like those used by Twyman et al. (2009), relative to categorical differences, may be advantageous because the scalar cues provide two relative dimensions for comparison (the walls vary in dot size and number of dots per wall). Because ordinal information can be mapped more readily onto spatial position than nominal information, mice may find the scalar cues to be more robust (Twyman et al., 2009). Additionally, the relative encoding and preferential use of featural and geometric cues by mice is strongly influenced by experience. Young and adult mice housed in circular environments (no informative geometric cues) and trained in the rectangular arena task showed a strong reliance on featural cues, but did not incidentally encode the arena's geometric properties (Twyman, Newcombe, \& Gould, 2013). However, young and adult mice housed in rectangular environments also showed a strong reliance on featural cues, but they incidentally encoded geometry. Thus, the properties of the rearing or housing environment of the mice strongly influenced which cues were used when reorienting. Even short-term experience with cue stability and reliability, gained from experimental training, has been shown to influence cue use. Mice trained in a feature-rich environment, but experiencing featural cue instability, showed an incidental encoding of geometry (Leonard et al., 2018), whereas mice that did not experience featural cue instability during training did not incidentally encode geometry. Results from these studies support that the encoding of featural and geometric information by mice is highly malleable, and experience strongly influences the encoding of these cues.

Mice also show changes in the encoding of featural and geometric cues across the life span. During one of the first studies to examine how the use of cues for reorientation changes with age, adult (3-5 months) and aged (20-21 months) mice were trained to search for a hidden escape platform located in one corner of a rectangular water maze (Fellini, Schachner, \& Morellini, 2006). A single, patterned featural cue was positioned on one of the interior short walls of the maze adjacent to the escape platform. After establishing reliable search behaviour with the feature present, this distinctive cue was removed to examine whether the mice incidentally encoded the geometric cues during training. Adult, but not aged, mice showed an ability to use the geometric properties of the environment, supporting that the process of aging disrupts the incidental encoding of geometry. This result suggests that mice may be a good candidate species for the study of age-related changes in cue use during reorientation, as during adulthood both cue types are encoded, but with age the encoding of geometry is disrupted as has been reported for healthy aging adult humans (Moffat, 2009; Moffat \& Resnick, 2002; Siemens \& Kelly, 2017). However, as few studies have been conducted to examine how aging influences the encoding of geometry, further studies are needed to corroborate these findings (Siemens \& Kelly, 2017).

Many neurodegenerative disorders affect an individual's ability to successfully orient. Alzheimer's disease (AD) is perhaps one of the most well-document disorders known to cause substantial decrements of spatial ability (Monacelli, Cushman, Kavcic, \& Duffy, 2003; Serino, Cipresso, Morganit, \& Riva, 2014). Individuals with $\mathrm{AD}$ often experience feelings of being lost or disoriented, and these symptoms can appear early in the disease's trajectory (Lithfous, Dufour, \& Despres, 2013). Orientation is a complex cognitive process involving many components such as memory, visual cue use, and attention (Wolbers \& Hegarty, 2010). Different mouse models of AD have been developed, with each typically manifesting different neuropathologies associated with AD (Sasaguri et al., 2017), to identify how these brain changes influence different cognitive functions. One well-studied model is the $3 \times T$ Tg-AD strain $(3 \times T g)$, which is a triple-transgenic model that expresses many of the neurological changes characteristic of AD. This model is particularly relevant, as 3xTg mice possess three mutations for genes associated with AD: APP Swedish, MAPT P301L, and PSEN1 M146V (Oddo et al., 2003). The brains of $3 \times \mathrm{Tg}$ mice exhibit the characteristic $\mathrm{A} \beta$ plaque 
deposition, selectively located within the Alzheimer's-relevant hippocampus and cerebral cortex, as early as 3-4 months (Clinton et al., 2007). The extent of degradation progresses across the life span, and neurofibrillary tangles, caused by the pathological hyperphosphorylation of tau proteins, occur around the ages of 12-15 months. Coincident with these neurological changes, marked behavioural and cognitive deficits have been documented. In particular, $3 \times \mathrm{xTg}$ mice, relative to controls, show increased anxiety and elevated startle responses (Sterniczuk, Antle, LaFerla, \& Dyck, 2010), as well as deficits during tests of object recognition and inhibitory control (Filali et al., 2012). Despite these deficits, 3xTg mice display no impairments on tests of reflex, nesting behaviour, or visuomotor skills (Filali et al., 2012), and show comparable visual acuity to C67BL/6 mice (King, Wong, \& Brown, 2018), indicating specificity in the way that the transgenes influence the mouse's behaviour. Importantly, $3 \times \mathrm{Tg}$ mice also show poorer performance on tests of reference and working memory (Stevens \& Brown, 2015), as well as tests of spatial memory using the Barnes maze (Stover, Campbell, Van Winssen, \& Brown, 2015). Although 3xTg mice show impairments in the use of distal cues as landmarks for spatial reorientation (Stimmell et al., 2019), it is not known what properties of these landmarks, features or geometry, fail to be encoded by $3 \times \mathrm{xTg}$ mice.

The goal of the present study was twofold. Firstly, we wanted to determine whether the encoding of environmental geometry is degraded with age, and secondly, we wanted to determine whether $3 \times \mathrm{Tg}$-AD mice show different impairments in the use of featural and geometric cues for reorientation, compared with healthy wild-type mice.

\section{Method}

\section{Subjects}

Mice were divided into four groups based on strain (3xTg or C57BL/6) and age (5 months or 15 months, henceforth referred to as "adult" and "aged," respectively). The age for the adults was chosen because $3 \times \mathrm{Tg}$ mice start showing $\mathrm{A} \beta$ plaques at 3-4 months, and thus may already show the beginnings of cognitive signs of impairment, although results regarding precise onset of cognitive impairments are mixed (e.g., Sterniczuk et al., 2010; Stover et al., 2015). Thus, the rationale for selecting this age group was to ensure the $3 \times \mathrm{Tg}$ mice were in the adult stages (as cue use is known to change with development), and the presence of at most mild cognitive impairments would be expected. The adult groups for both strains were composed of 10 mice each, whereas the aged groups were composed of seven and six mice for the $3 \mathrm{xTg}$ and $\mathrm{C} 57 \mathrm{BL} / 6$ groups, respectively (the difference in sample size was due to attrition of mice at older ages). All mice were experimentally naive, and obtained through The Jackson Laboratory. The mice were housed individually (cages of 29 -cm length $\times 18.5$-cm width $\times 13$-cm height) within a colony room with a 12-hour light-dark period (light onset at 0700). These cages contained objects for enrichment, such as, a plastic shelter and Nylabone brand dog bone. Ad libitum access to Prolab® RMH 3000 food pellets and water was provided at all times. All housing and experimental procedures were approved by the University of Manitoba Animal Care Committee (protocol \# F15-027) and complied with the guidelines of the Canadian Council on Animal Care.

\section{Materials}

The experimental arena consisted of a rectangular arena filled with water (in the fashion of a Morris water maze). Identical white sheets of corrugated plastic were attached to the inside of the arena to form the walls ( $90-\mathrm{cm}$ length, $42-\mathrm{cm}$ width, and $55-\mathrm{cm}$ height; see Fig. 1). Feature information was presented on removable laminated panels that were attached to the interior walls of the arena with Velcro strips. Each feature panel completely covered either a long wall or a short wall. Two panels with different patterns were used: one consisting of rows of large diamonds (each diamond $27-\mathrm{mm}$ width $\times 37$ $\mathrm{mm}$ height), and the other consisting of rows of small clubs (each club 8 -mm width $\times 8$-mm height). These cues were selected because previous research has reported that mice more readily reorient in the presence of featural cues differing along a scalar dimension, rather than features that differ categorically (Twyman et al., 2009). Our cues differed along a scalar dimension, and categorically, to ensure stimulus discrimination. The featural cues were configured such that one long wall and an adjacent short wall displayed the diamond pattern, whereas the opposite two walls displayed the club pattern (see Fig. 2a).

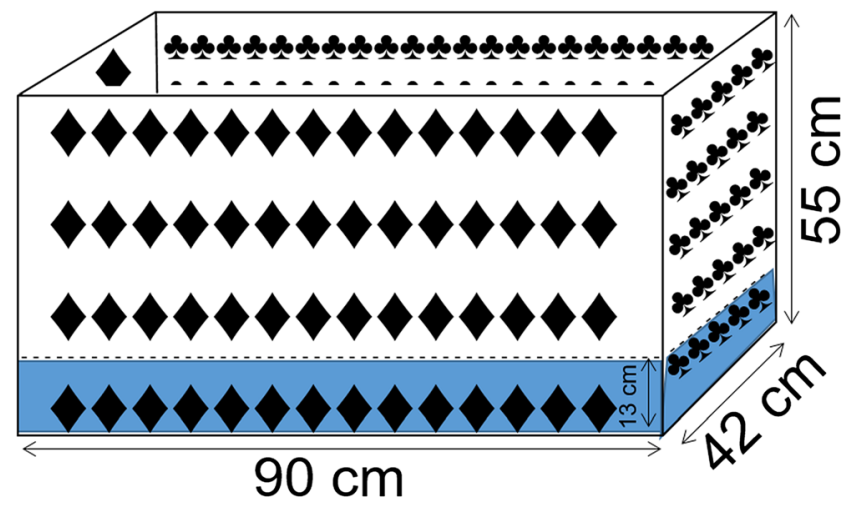

Fig. 1 Schematic of the rectangular water maze with the featural configuration used during training. Both feature patterns (diamonds or clubs) were presented on a short and long walls. Dashed line and shading indicates water level depth at $13 \mathrm{~cm}$. The schematic is for illustrative purposes, and not drawn to scale 

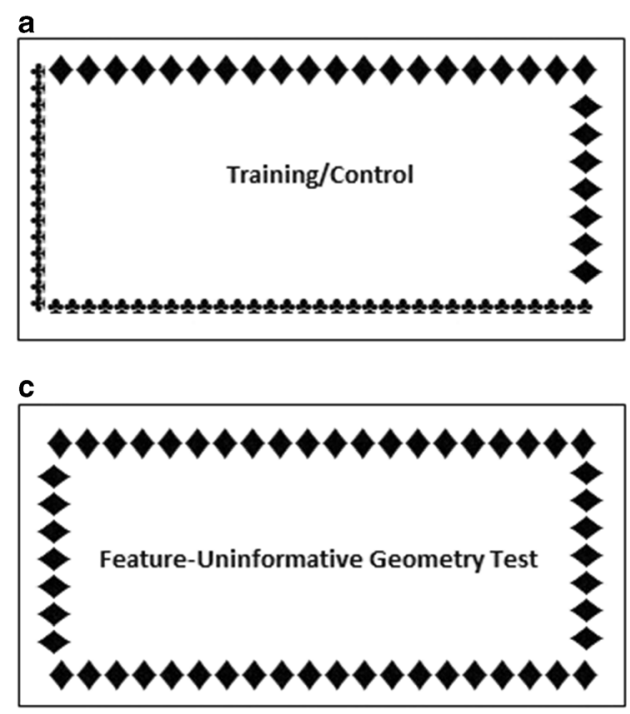

b

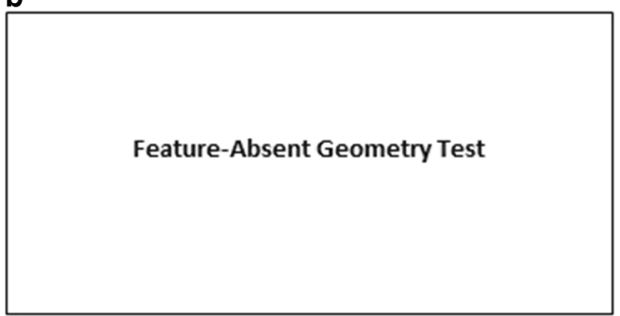

d

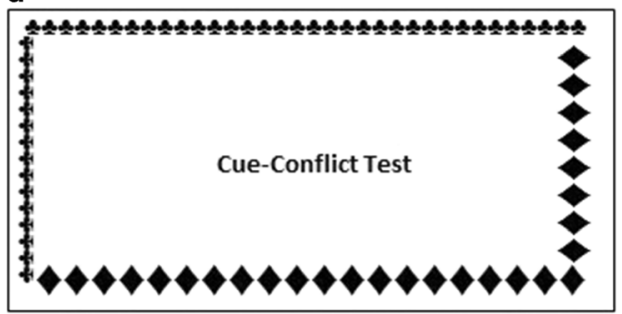

Fig. 2 The configuration of feature panels during (a) training and control trials, (b) feature-absent test trials, (c) feature-uninformative test trials, and (d) cue-conflict test trials. Schematic are for illustrative purposes only, and are not drawn to scale

One white ceramic tile $(10 \mathrm{~cm} \times 10 \mathrm{~cm})$ was used as a removable platform for the mice to exit from the water, and its height could be adjusted based on the experimental phase. This platform was positioned in one of the four corners of the arena, $5 \mathrm{~cm}$ away from the nearest short and long wall, with the corner location depending on training group. The space between the platform and the walls allowed the mouse to swim without accidental contact with the platform, preventing encounters through thigmotaxis (the tendency for rodents to stay close to the walls of an open space). The location of the platform remained consistent across training for each individual mouse.

The arena was filled with water $\left(13-\mathrm{cm}\right.$ depth, $20^{\circ} \mathrm{C} \pm 1^{\circ} \mathrm{C}$, replaced daily) made opaque with the addition of instant skim milk powder (Dairytown Processing Ltd.). The opacity of the water eliminated the use of the platform itself as a visual cue, and enhanced the color contrast for the motion-tracking software. To prevent the use of external visual cues, the arena was surrounded by white curtains. To prevent the use of external auditory cues, a radio was played in the experimental room, and its location within the room was changed after every second trial. A Javelin video camera was suspended over the arena, which was connected to a computer operating the BiObserve Viewer motion-tracking software used to record all trials.

\section{General procedures}

Mice were individually transported to the experimental room in an empty holding cage identical to their home cages. Before each trial, the mouse was removed from the holding cage and placed into an opaque circular container. The mouse was then disoriented by rotating the container on a stool six rotations clockwise and six counterclockwise (12 rpm) to remove the potential use of inertial cues. The experimenter then completed a random walk to arrive at the position where the mouse would be placed by hand into the arena, which was along the center of one of the arena's walls. The start position was counterbalanced across the four possible positions within each daily session. The mouse was placed into the arena facing the predetermined wall. The mouse was permitted to swim until it reached the platform, or 60 seconds had elapsed, whichever occurred first. Once a trial was completed, the mouse was removed from the arena and returned to its holding cage for an intertrial interval of 10 minutes. A $150 \mathrm{~W}$ heat lamp was placed above the holding cage to provide additional warmth (Iivonen, Nurminen, Harri, Tanila, \& Puoliväli, 2003).

\section{Training Phase 1}

At the onset of the experiment each mouse was assigned a reinforced corner (i.e., the corner in which the platform would be located) as either the corner containing diamonds or clubs on both adjacent walls (the two corners subtended by walls of different patterns were not used as reinforced corners; see Fig. 2a). Training Phase 1 consisted of four trials per session, with one session per day. During the first day of training, the platform remained visible approximately $1-\mathrm{cm}$ above the surface of the water. As mice reliably located the platform, it was gradually lowered over sessions until it was no longer visible (approximately $2-\mathrm{cm}$ below the surface). If a mouse was unable to locate the platform within 60 seconds, it was guided to the platform by the experimenter's hand. Once the mouse was on the platform, it was allowed to remain there for 30 seconds before being removed. To ensure mice were unable to use cues 
external to the arena, the feature panels were rotated $180^{\circ}$ within the arena, and the radio and rotating stool were moved to a different location in the experimental room. To advance to the next training phase, a mouse was required to swim to the fully submerged platform in less than 20 seconds during each trial, for two consecutive sessions.

\section{Training Phase 2}

Training Phase 2 was identical to Training Phase 1, with the exception that one of the trials (but never the first one) was replaced with a platform-absent trial, wherein the platform was removed from the arena. During these platform-absent trials, the mouse was allowed to swim for 60 seconds before being removed from the arena. The platform-absent trials were instituted to ensure that the mice were familiar with trials without a platform present. To advance to the testing phase, mice were required to reach the platform in less than $20 \mathrm{sec}-$ onds during all three platform-present trials and spend at least twice as much time in the quadrant with the platform as in the second most visited quadrant, for two consecutive sessions. If a mouse was unable to locate the platform within 60 seconds during one of the three trials for two consecutive sessions, it was moved back to Training Phase 1 .

\section{Testing phase}

The testing phase consisted of 16 testing sessions, with eight trials per daily session. The odd-numbered trials were identical to training trials with respect to the feature configuration, and the platform was present. The even-numbered trials were either control or test trials. Control trials were identical to the platform-absent trials from Training Phase 2. Test trials were also conducted with the platform absent, but additional manipulations were made to the featural information on the walls of the arena. During both the control and test trials, the mouse was permitted to swim for 60 seconds before being removed from the arena by the experimenter. During each session, the mouse received one control trial and three test trials (one trial of each test condition: feature-absent geometry, featureuninformative geometry, and cue conflict).

Feature-absent geometry test During the feature-absent geometry test, the diamond and club featural panels were removed and were replaced with uniform white panels (see Fig. $2 b$ ). The feature-absent geometry test examined whether the mice had incidentally encoded the geometric properties of the environment during training. If the mice had encoded the geometry, we predicted they would preferentially search for the platform at the two corners sharing the same geometric properties as their trained corner (geometrically correct corners); that is, they would show rotational errors. However, if the mice had not encoded geometry, we predicted they would divide their search behaviour among all four corners.

Feature-uninformative geometry test During the featureuninformative geometry test, the feature panels were replaced with four identical panels displaying the reinforced feature panels for a particular mouse (e.g., a mouse with the platform located at the corner with both the long and short wall displaying the diamond pattern would have all four walls displaying the diamond pattern during the feature-uninformative geometry test; see Fig. 2c). The feature-uninformative geometry test examined whether the presence of the reinforced features during testing facilitated the use of geometry, even though the featural information was not itself informative (as the features were identical across all walls), as has been shown for other species (Kelly, 2010). It is possible that the mice encoded the geometry of the training arena, but the feature-absent arena might look so different that the mice treat it as a new environment. The feature-uninformative geometry test retained elements of familiarity with the trained arena by making the viewpoints provided from within the test arena more similar to the trained arena, but still required the mice to reorient based on the geometric cues. As in the feature-absent geometry test, if the mice had encoded the geometry, we predicted they would preferentially search for the platform at the two corners sharing the same geometric properties as their trained corner (geometrically correct corners); they would show rotational errors. However, if the mice had not encoded geometry, we predicted they would divide their search behaviour among all four corners.

Cue-conflict test During the cue-conflict test, the feature panels on the short walls of the arena were swapped with each other (this functionally produced a counterclockwise rotation of each feature panel; see Fig. 2d). This modification created a conflict between the feature and geometric information as to the expected location of the platform, and allowed for the evaluation of whether the mice would weigh featural or geometric cues more heavily when reorienting. If the mice weighed the featural cues more heavily, we would expect them to search initially at the corner containing the featural cue, whereas if geometric cues were weighed more heavily, the mouse would initially search at one of the two geometrically correct corners.

\section{Statistical analyses}

To examine the accuracy of search behaviour, analyses were conducted on the proportion of trials during which a mouse swam first to each corner. Using the BiObserve tracking software, equivalent areas (herein referred to as "corners") were defined at each of the four corners corresponding to the platform's size and relative location. A first choice was considered to be the corner the mouse swam into first when 
placed into the arena. Corner choice, rather than swim speed, was use as previous research has shown that $3 \mathrm{x}-\mathrm{Tg}$ mice express hyperactivity in similar tasks, suggesting that measures based on swim speed may be less sensitive for detecting AD-related cognitive deficits (Baeta-Corral \& GiménezLlort, 2015). Additionally, swim speed may be more susceptible to age-related motoric decline. An ANOVA with between-subjects measures (strain: $3 \times \mathrm{xTg}$ or $\mathrm{C} 57 \mathrm{BL} / 6$ and age: adult or aged) was conducted, with the proportion of first choices to a specific corner as the dependent measure for the control trials. For each testing condition, an ANOVA with between-subjects measures (strain: $3 \times \mathrm{Tg}$ or C57BL/6 and age: adult or aged) was conducted, with the proportion of first choices to a specific corner as the dependent measure. To evaluate whether performance differed from chance, onesample $t$ tests were conducted. To evaluate whether the distribution of choices differed between two corners, or among several corners, paired-samples $t$ tests or chi-square tests were conducted, respectively. Significant alpha level was set to $\leq 0.05$.

\section{Results}

\section{Learning rate}

We found significant differences in the number of sessions required to learn the task for the strain and age of the mice, Strain, $F(3,32)=221.22, p<.001$; Age, $F(3,32)=165.42, p$ $<.001$; a Strain $\times$ Age interaction, $F(1,32)=7.71, p=.009$. Specifically, the adult $\mathrm{C} 57 \mathrm{Bl} / 6$ mice required fewer trials to meet the learning criteria $(M=24.8 \pm 1.4)$ than each of the other groups [aged C57Bl/6 $(M=46 \pm 2)$ mice, adult $3 \times \mathrm{Tg}(M$ $=48.8 \pm 1.5)$, or aged $3 \times \operatorname{Tg}$ mice $(M=62.4 \pm 0.65)]$, which did not differ from each other. All groups reached the same criterion prior to advancing to the testing phase.

\section{Control trials}

To examine whether there were differences in the accuracy of the mice during the testing phase, an ANOVA was conducted on the proportion of first choices to the trained corner during control trials across the testing conditions. There was no significant effect of Strain, $F(3,34)=0.03, p=.86$; Age, $F(3,32)$ $=0.03, p=.86$; and no significant Strain $\times$ Age interaction, $F(3,32)=3.19, p=.08$. Furthermore, all groups of mice were locating the correct corner significantly better than expected by chance (chance $=0.25 ; M \mathrm{~s}=0.74 \pm 0.03$, and $0.67 \pm 0.04$, for the adult and aged $\mathrm{C} 57 \mathrm{Bl} / 6$ and $M \mathrm{~s}=0.69 \pm 0.03$ and 0.76 \pm 0.05 for the adult and aged $3 \times \mathrm{xg}$ mice, respectively; onesample $t$ test: all $t \mathrm{~s}>10.84$, all $p$ s $<.001$ ).

\section{Feature-absent geometry test}

To examine whether age or strain affected whether the mice showed an encoding of the geometry of the arena, an ANOVA, with between subjects factors of strain and age, was conducted on the proportion of first choices to the geometrically correct corners made by the mice during the feature-absent geometry test. There was no significant effect of Strain, $F(3,32)<0.01, p=.99$, or Age, $F(3,32)=0.76, p=$ .39 , and no significant Strain $\times$ Age interaction, $F(1,32)=$ $1.34, p=.26$. Indeed, reorienting in the featureless environment proved difficult for all of the groups of mice, as on average none of the groups went first to the geometrically correct corners more than would be predicted by chance (chance $=0.50 ; M \mathrm{~s}=0.54 \pm 0.04,0.43 \pm 0.07$, for the adult and aged $\mathrm{C} 57 \mathrm{Bl} / 6$ and $M \mathrm{~s}=0.49 \pm 0.06,0.50 \pm 0.04$ for the adult and aged $3 \times T g$ mice respectively; one-sample $t$ test: all $t \mathrm{~s}$ $<1.03$, all $p \mathrm{~s}>.33$ ). Indeed, the groups distributed their choices equally among the four corners, supporting that they were unable to use the environmental geometry (or any external cues) to guide orientation (all $\chi^{2}<36.13$, all $p \mathrm{~s}>.11$ ).

\section{Feature-uninformative geometry test}

To examine whether the presence of uninformative features facilitated the use of geometry, an ANOVA was conducted on the proportion of first choices to the geometrically correct corners made by the mice during the feature-uninformative geometry test. We found no significant effect of Strain, $F(3$, $32)=2.93, p=.10$, and no significant Strain $\times$ Age interaction, $F(1,33)=0.00, p=.96$. However, the main effect of Age was significant, $F(3,32)=3.96, p=.05$. One-sample $t$ tests were conducted for each age group, comparing the proportion of first choices with the geometrically correct corners against chance $(0.50)$. These tests revealed that some, but not all, groups had encoded geometry. Adult mice from both strains swam first to the geometrically correct corners more than would be predicted by chance, $M=0.71 \pm 0.03, t(9)=6.81$, $p<.01$, and $M=0.65 \pm 0.03, t(9)=4.64, p<.01$, for adult $\mathrm{C} 57 \mathrm{Bl} / 6$ and $3 \mathrm{xTg}$ mice respectively, whereas neither strain of aged mice showed such an encoding of geometry, $M=0.64 \pm$ $0.07, t(5)=2.01, p=.10$, and $M=0.58 \pm 0.04, t(6)=1.81, p=$ .10 , for aged $\mathrm{C} 57 \mathrm{~B} 1 / 6$ and $3 \times \mathrm{Tg}$ mice, respectively. Although the mean accuracy of the aged C57B1/6 (0.64) was similar to that of the adult $3 \times \mathrm{xTg}$ mice $(0.65)$, the aged $\mathrm{C} 57 \mathrm{Bl} / 6$ failed to reach statistical significance at a group level due to greater interindividual differences in performance. Indeed, three of the aged $\mathrm{C} 57 \mathrm{Bl} / 6$ mice performed at chance level $(M=0.50$, 0.56 , and 0.50 , respectively), whereas the other three performed above chance level $(M=0.69,0.63$, and 0.94 , respectively). This interindividual difference was not seen within the adult $3 \times$ Tg group $(M s=0.50,0.56,0.56,0.63,0.63,0.63$, $0.68,0.68,0.75,0.82)$. The increase in interindividual 
differences with age was expected, and may be indicative of low-performing and high-performing aging, as reported in studies of human cognition (Cabeza, Anderson, Locantore, \& McInstosh, 2002).

\section{Cue-conflict test}

To examine the distribution of choices during the cue-conflict tests, an ANOVA was conducted on the proportion of choices made to the featurally correct corner. There was a main effect of Strain, $F(3,32)=5.33, p=.03$, with $\mathrm{C} 57 \mathrm{Bl} / 6$ mice $(M=$ $0.31 \pm 0.05)$ choosing the corner containing the correct feature more often than the $3 \times \mathrm{Tg}$ mice $(M=0.19 \pm 0.05)$. The main effect of Age was significant, $F(3,32)=8.21, p=.01$, with adult mice $(M=0.31 \pm 0.04)$ choosing the corner containing the correct feature more than aged mice $(M=0.16 \pm 0.05$; see Fig. 3 for proportion of choices to all four corners of the arena for each strain). The aged mice, however, preferentially chose the geometrically correct corner nearest to the displaced feature, $M=0.44 \pm 0.07, t(5)=2.50, p=.05$, and $M=0.45 \pm$ $0.08, t(6)=2.55, p=.03$, for aged $\mathrm{C} 57 \mathrm{Bl} / 6$ and $3 \times \mathrm{Tg}$ mice, respectively. There was no significant Strain $\times$ Age interaction, $F(1,33)=0.14, p=.72$. Additionally, the adult $\mathrm{C} 57 \mathrm{Bl} / 6$ chose the single corner which was incorrect according to both featural and geometric cues less than expected by chance, $M=$ $0.07 \pm 0.03, t(9)=6.26, p<.01$, indicating that they were avoiding the corner which was not associated with any of the previously reinforced cues, whereas the adult $3 \times \mathrm{Tg},(M=0.17$ $\pm 0.05)$, aged $3 \times \operatorname{Tg}(M=0.22 \pm 0.06)$, and aged $\mathrm{C} 57 \mathrm{Bl} / 6(M=$ $0.15 \pm 0.06)$, did not (all $t \mathrm{~s}<1.69$, all $p \mathrm{~s}>0.08)$.

\section{Discussion}

The current study was designed to determine whether the ability to encode environmental geometry degrades with age, and whether 3xTg-AD mice show differential impairment in the use of featural and geometric cues for reorientation, compared with healthy wild-type mice. Our results showed that adult and aged mice had difficulty encoding geometry, when presented in a "featureless" environment (feature-absent geometry test). However, by providing seemingly uninformative features, the adult mice of both strains were able to reorient based on geometry, but the aged mice could not, supporting that aging affects the encoding of geometry. We also found results suggesting that the two strains of aged mice were differentially impaired. The aged C57B1/6 mice showed interindividual differences in the encoding of geometry when uninformative features were provided (features-uninformative geometry test), with some individuals being able to use geometry successfully. However, the presence of uninformative features did not facilitate the ability to use geometry for the aged 3xTg-mice.

Previous research has also shown that adult mice do not readily use geometry for reorienting in a "featureless" environment. However, when reared in geometrically enriched environments, mice have been shown to use geometric cues

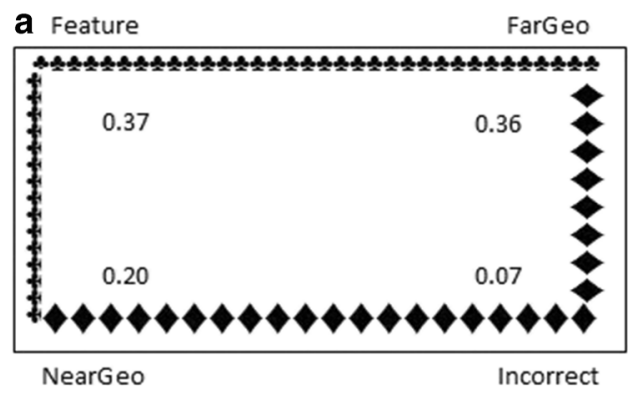

Adult: $\mathrm{C} 57 \mathrm{BI} / 6$

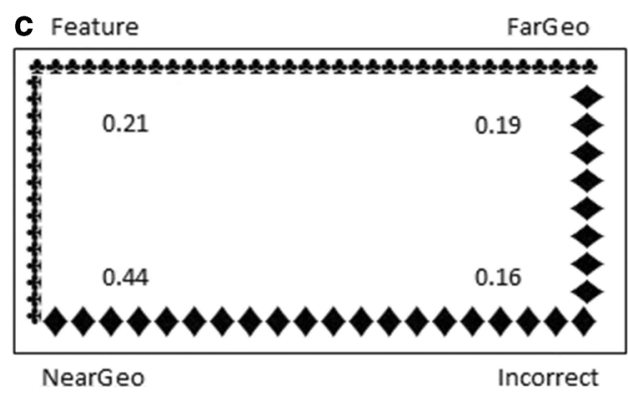

Aged: $\mathrm{C57BI} / 6$

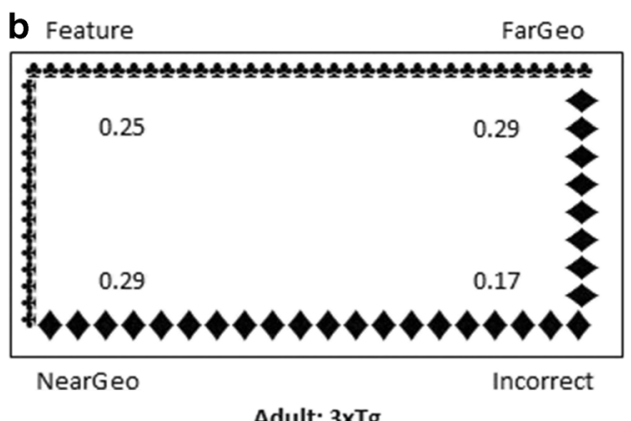

Adult: $3 \times 1 g$
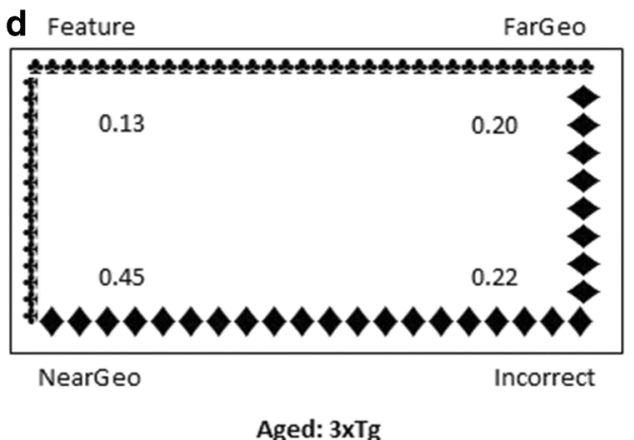

Fig. 3 Proportion of first choices made to each corner during the cue-conflict test. Schematic representations are for illustrative purposes only, and are not drawn to scale 
(Twyman et al., 2013). Twyman and colleagues showed that young and adult mice housed in rectangular environments used environmental geometry during a reorientation training paradigm, whereas mice housed in circular environments did not. This suggests that the ability for mice to reorient using geometry may need experience with such cues. Similarly, using the same reorientation paradigm as the current study, albeit in an appetitive context, Leonard et al. (2018) trained mice to search for food in one corner of a rectangular arena with similar feature panel configurations. The mice were then tested using discrete testing blocks, with some mice receiving tests with the features removed (geometry testing) followed by tests of cue-conflict, and others receiving the counterbalanced order. Mice in that study did not show an encoding of geometry when tested with the white-walled arena unless they had preceding experience with featural cue instability, again suggesting that experiential factors are important in the mouse's ability to use geometric cues. Mice in the present study were presented with interleaved testing trials, therefore none had extensive experience with featural cue instability before their experience in the feature-absent geometry test. Thus, without either the experience with featural cue instability, or exposure to enriched geometric environments, mice appear to have difficulty encoding geometric cues when featural cues are stable and reliable.

One possible reason for the difficulty observed in the present study may be due to the nature of the featural cues used. We used continuous featural panels displayed across the entirety of the four walls. This creates a greater, and likely more noticeable, visual change between the training and featureabsent geometry testing environments than would exist if the featural cues were smaller discrete objects located in the corners. During studies using discrete objects as features, the walls of the arena do not change during tests of geometric encoding, only the features in the corners. Under such circumstances the encoding of the geometric information learned during training might be more readily transferred to the feature-absent arena, as the visual difference between training and testing is minimized. Similarly, previous reports of incidental geometric encoding by mice in a Morris water maze may have benefited from the use of a single featural panel along one wall, with the remaining walls uniformly white (Fellini et al., 2006). During that study, when the featural panel was removed, the visual difference between training and testing would have been again minimized. The visual disparity between the training environment and the featureabsent environment in the present study may have caused mice to treat the feature-absent environment as a completely novel environment, and although they may have incidentally encoded the environmental geometry during training, they did not transfer this information to the "new" environment. Thus, poor performance during the feature-absent geometry test, relative to the feature-uninformative geometry test, is likely not due to a failure of geometric encoding, at least by the adult mice, but rather a failure in retrieval or use of those cues.

When the test of geometric encoding included the presence of seemingly uninformative features, as in the featureuninformative geometry test, both adult $\mathrm{C} 57 \mathrm{Bl} / 6$ and $3 \times \mathrm{Tg}$ mice showed a preference to search in the corners containing the same geometry as their trained corner. During this test, the presence of identical feature patterns along all four walls made the testing environment more closely resemble the training environment. From a view-matching perspective, the geometrically correct corners during the feature-uninformative geometry test would have been visually identical to the trained corner for which the mouse had been reinforced, according to both geometric and featural cues (Pecchia \& Vallortigara, 2010; Wystrach, Cheng, Sosa, \& Beugnon, 2011).

An alternative explanation for these results might be generalization decrement - the reduction in conditioned responding that occurs with greater magnitude with increasing differences between training and testing stimuli (Pearce, 1987). The featural change between the training and testing environments was greater for the feature-absent geometry test compared with the feature-uninformative geometry test, as the former environment contained none of the featural cues present during training, whereas the latter contained the reinforced feature. Thus, having the featural cues present (although uninformative) may have facilitated the use or retrieval of the geometry learned during training by reducing the magnitude of the difference between the training and testing environments.

Finally, the specific patterning of the featural cues may be the reason we found differences between the two geometry tests. The mere presence of patterned elements on the wall, as opposed to a blank white wall, may have increased the salience of each wall length or differentiation of the corners. This additional cue may have helped the mice distinguish the relative lengths of the walls. Future studies could distinguish between these alternatives by employing a geometry test in which uninformative features are displayed across the four walls, but using features that were not used during testing (novel features). This approach would allow one to assess whether it is either the familiarity of the features (from training) or the mere presence of the features for perceptual differentiation that supported the geometric encoding by the adult mice.

With respect to the aged mice of both strains, we found no significant differences between strains, and neither strain showed an ability to use the geometric cues during either the feature-absent geometry or the feature-uninformative geometry test at the group level. This corroborates previous research with mice, which found that aged mice were unable to incidentally encode environmental geometry when trained with stable and robust featural cues (Fellini et al., 2006). However, several individual aged mice of both strains did show evidence of having encoded the geometric cues. This suggests the aging process affects individuals differently, and 
that some individuals retain the ability to use geometry to reorient, even into old age. Thus, mice may show interindividual differences similar to that reported for humans, in that some mice may be low and high performing (Cabeza et al., 2002). Future research should employ a greater sample size to better examine these interindividual differences to further our understanding of which factors on an individual level result in age-related cognitive decline in this important animal model.

During the cue-conflict test, only adult $\mathrm{C} 57 \mathrm{Bl} / 6$ mice preferentially went to the corner containing the displaced featural cue, relative to chance. Amongst the other groups, mice either showed no selectivity in choice between any of the corners (as did the adult $3 \times \mathrm{Tg}$ mice) or showed a preference for the geometrically correct corner nearest to the displaced featural cue (aged mice of both strains). This latter result is particularly intriguing, as neither group of aged mice showed an encoding of geometry, yet during the cue-conflict test, the aged mice appeared to be choosing the corner that was a compromise between featural and geometric cues. During the cue-conflict test, the geometrically correct corner nearest to the featural corner ("NearGeo" in Fig. 3) contained one of the features associated with the platform during training. Thus, this corner is correct according to geometry and partially correct according to features (one of the two features in the corner was correct). Although this appears to conflict with results from the feature-absent and feature-uninformative tests, during which they seemed unable to use geometric cues, this discrepancy might also be explained through generalization decrement. During the cue-conflict test, all of the featural and geometric cues provided during training were present during the cue-conflict test, albeit in a different configuration. This contrasts with the feature-uninformative test, for which only the reinforced feature was present, and the feature-absent for which all features were absent. Thus, the differential performance between the adult $\mathrm{C} 57 \mathrm{Bl} / 6$ and aged mice of both strains during the cue-conflict test may best be interpreted as a difference in the relative salience of the featural and geometric cues, or differences in attending to the respective cues (Buckley et al. 2015). However, why adult and aged mice would differ in how they attended to the featural and geometric cues remains unclear.

Featural and geometric cues are frequently considered as discrete visual cue types - an object is red (featural cue) and is located 100-cm north (geometric cue) of another-and these cues may be combined in different ways. However, research conducted by Stephen Lea and colleagues has shown that nonhuman animals may not separate cues in such an independent fashion, particularly if they do not impose a rule-based generalization on the visual cues in their environment (Maes et al., 2015). For instance, rats and pigeons trained to associate a certain outcome for two independently presented stimuli ("A" and "B"), but a different outcome for the compound of the two stimuli ("AB"), are unable to generalize to novel stimuli (e.g., "C" and "D" and "CD"). This result differs from that reported when human participants were examined, as they successfully transferred this "opposites rule" (Maes et al., 2015). Species differences in the integration or separation of cues may be particularly important when considering results from cue-conflict tests. Such tests are designed to assess the degree to which an animal may weigh the validity or reliability of the two cues as independent stimuli. However, an alternate interpretation may be that an animal associates the "view" with reinforcement, and in doing so integrate the featural and geometric cues. Such view matching has been suggested for a variety of species (Graham \& Cheng, 2009; Pecchia \& Vallortigara, 2010; for a review see Cheng et al., 2013). Relatedly, previous studies using multidimensional stimuli trained subjects to categorize stimuli based on individual elements (e.g., shape, pattern, colour) into two experimenterdefined categories and then subsequently required them to categorize stimuli composed of three elements. The multidimensional stimuli could have their elements spatially separated (appearing as an array of three items), or their elements spatially integrated into one single item. During cue-conflict testing trials, one of the elements of the multidimensional stimulus (e.g., its pattern) would be incorrect, resulting in the overall stimulus that was "one-off” from being correct. When the elements were spatially integrated, humans and pigeons (and gray squirrels) were more likely to categorize stimuli unidimensionally (i.e., using only one of the learned dimensions, for example "shape"), whereas when the dimensions were spatially separated, humans and pigeons categorized the stimuli based on their overall similarity to the trained multidimensional stimuli (Wills et al., 2009 also see Lea et al., 2009). These results may have important implications for understanding cue use for reorientation, particularly if the properties (or dimensions) of a stimulus are spatially integrated. Although studies have begun to examine the use of viewmatching strategy during reorientation, this topic has not yet been explored with mice.

To our knowledge the current study is the first to examine whether the encoding of geometry is differentially affected during healthy and degenerative aging processes in mice. Spatial working memory deficits, as well as the beginnings of $\mathrm{A} \beta$ deposition in the brain, have been observed in $3 \times \mathrm{Tg}$ mice as young as four months (Clinton et al., 2007). Thus, if the $\mathrm{A} \beta$ deposition was responsible for any deficits in the use of geometric (or featural) cues, we would should have seen strain differences in our 5-month-old adult mice, which we did not. Although we did find significant overall strain differences in the number of trials needed to initially learn the task.

Finally, during the current study, the features were presented as large, patterned surfaces, whereas previous studies showing impairments in the $3 x \mathrm{Tg}$ mice during a reorientation task used discrete landmarks as feature cues (Stimmell et al., 2019). However, previous research in other species has 
supported that surface-based features as well as features presented as discrete objects are readily used for reorientation (Lee \& Spelke, 2010). Thus, during the present study, it is unlikely that the mice had difficulty reorienting using the surface-based feature cues. However, many studies have supported that geometry is differentially encoded when presented as continuous surfaces (such as walled environments) or as a configuration of discrete objects, with the former encoded using relative metrics and the latter encoded using absolute metrics by several nonhuman species (Kelly \& Spetch, 2001; Spetch, Cheng, \& MacDonald, 1996; Spetch et al., 1997). Indeed, accumulating behavioural and neurological evidence suggests that three-dimensional extended boundaries play a unique role in spatial navigation (Lee, 2017). Future studies are needed to better understand whether the presentation of discrete or continuous featural and geometric cues is differentially affected by aging processes.

The current study was designed to determine whether the ability to encode environmental geometry degrades with age, and whether $3 \times \mathrm{Xg}-\mathrm{AD}$ mice show different impairment in the use of featural and geometric cues for reorientation, compared with healthy wild-type mice. Our results showed that adult and aged mice had difficulty encoding geometry, when presented in a "featureless" environment (features-absent geometry test), but the presence of uninformative features allowed the adult mice of both strains to reorient, but not the aged mice, suggesting that age does affect the encoding of geometry. However, we also found results suggesting that the two strains of aged mice were differentially impaired. The aged $\mathrm{C} 57 \mathrm{Bl} / 6$ mice showed interindividual differences in the encoding of geometry when uninformative features were provided (features-uninformative geometry test), with some individuals being able to use geometry successfully. However, the presence of uninformative features did not facilitate the ability to use geometry for the aged 3xTg-mice.

In summary, the current study investigated whether the encoding of geometry during a reorientation task differed for wild-type mice and a transgenic mouse model for $\mathrm{AD}$, during adulthood and advanced age. We found that advanced age was the principal factor in the failure to incidentally encode geometry. However, the strains did show important interindividual differences - with aged individuals of the wild-type strain showing geometric encoding, whereas this was not the case with the aged $\mathrm{AD}$ mice. Furthermore, we found that the $\mathrm{AD}$ mice, regardless of age, required additional training to learn the initial task. Although it is well-established that spatial ability declines with advanced age, our results provide an important insight. Geometry is thought to be the foundation upon which featural cues are mapped (Cheng, 1986). Our study supports that with age this foundation degrades, clarifying how individuals can become disoriented in featurally familiar environments. Showing that mice may share similar degradation of geometric-based reorientation is a critical step in validating the mouse as an important animal model for studies of age-related decline in spatial cognition.

Author note This research was supported by a Natural Science and Engineering Research Council of Canada (NSERC) Discovery grant to D.M.K. (RGPIN/4944-2017). We thank Trevor Aquin for assistance with data analysis. All authors declare no conflict of interest. We wish to thank the anonymous reviewers for their insightful suggestions.

Open practices statement The data from this experiment will be provided as supplementary material upon publication. The experiment presented here was not preregistered.

\section{References}

Baeta-Corral, R., \& Giménez-Llort, L. (2015). Persistent hyperactivity and distinctive strategy features in the Morris water maze in 3xTg$\mathrm{AD}$ mice at advanced stages of disease. Behavioural Neuroscience, 129(2), 129-137. https://doi.org/10.1037/bne0000027

Bilkei-Gorzo, A. (2014). Genetic mouse models of brain ageing and Alzheimer's disease. Pharmacology and Therapeutics, 142(2), 222-257. https://doi.org/10.1016/j.pharmthera.2013.12.009

Buckley, M.G., Smith, A.D., \& Haselgrove, M. (2015).Learned predictiveness training modulates biases towards using boundary or landmark cues during navigation. The Quarterly Journal of Experimental Psychology, 68(6), 1183-1202.

Cabeza, R., Anderson, N. D., Locantore, J. K., \& McInstosh, A. R. (2002). Aging gracefully: Compensatory brain activity in highperforming older adults. NeuroImage, 17(3), 1394-1402. https:// doi.org/10.1006/nimg.2002.1280

Cheng, K. (1986). A purely geometric module in the rat's spatial representation. Cognition, 23(2), 149-178. https://doi.org/10.1016/00100277(86)90041-7

Cheng, K., Huttenlocher, J., \& Newcombe, N. S. (2013). 25 years of research on the use of geometry in spatial reorientation: a current theoretical perspective. Psychonomic Bulletin \& Review, 20, 103354. https://doi.org/10.3758/s13423-013-0416-1

Cheng, K., \& Newcombe, N.S. (2005). Is there a geometric module for spatial orientation? Squaring theory and evidence. Psychonomic Bulletin \& Review, 12, 1-23. https://doi.org/10.3758/BF03196346

Clinton, L.K., Billings, L.M., Green, K.N., Caccamo, A., Ngo, J., Oddo, S., McGaugh, J.L., \& LaFerla, F.M. (2007). Age-dependent sexual dimorphism in cognition and stress response in the $3 \times \mathrm{Tg}-\mathrm{AD}$ mice. Neurobiology of Disease, 28, 76-82.

Fellini, L., Schachner, M., \& Morellini, F. (2006). Adult but not aged C57BL/ 6 male mice are capable of using geometry for orientation. Learning \& Memory, 13(4), 473-481. https://doi.org/10.1101/lm.259206

Filali, M., Lalonde, R., Theriault, P., Julien C., Calon, F., \& Planel, E. (2012). Cognitive and non-cognitive behaviors in the triple transgenic mouse model of Alzheimer's disease expressing mutated APP, PS1, and Mapt (3xTg-AD). Behavioural Brain Research, 234, 334 342. https://doi.org/10.1016/j.bbr.2012.07.004

Graham, P., \& Cheng, K. (2009). Ants use the panoramic skyline as a visual cue during navigation. Current Biology, 19, 935-937. https:// doi.org/10.1016/j.cub.2009.08.015

Hardy, J., \& Selkoe, D.J. (2002). The amyloid hypothesis of Alzheimer's disease: Progress and problems on the road to therapeutics. Science, 297(5580), 353-356. https://doi.org/10.1126/science.1072994

Iivonen, H., Nurminen, L., Harri, M., Tanila, H., \& Puoliväli, J. (2003). Hypothermia in mice tested in Morris water maze. Behavioural Brain Research, 141, 207-213.

Kelly, D. M. (2010) Features enhance the encoding of geometry. Animal Cognition, 13(3), 453-462. 
Kelly, D. M., \& Spetch, M. L. (2001). Pigeons encode relative geometry. Journal of Experimental Psychology: Animal Behavior Processes, 27(4), 417-422. https://doi.org/10.1037/0097-7403.27.4.417

Kelly, D. M., Spetch, M. L., \& Heth, D. C. (1998). Pigeons' (Columba livia) encoding of geometric and featural properties of a spatial environment. Journal of Comparative Psychology, 112(3), 259-269. https://doi.org/10.1037/0735-7036.112.3.259

King, J. L., Wong, A. A., \& Brown, R. E. (2018). Age-related changes in the spatial frequency threshold of male and female $3 \times \mathrm{Tg}-\mathrm{AD}$ mice using OptoMotry. Journal of Alzheimer's Disease, 62(2), 591-596. https://doi.org/10.3233/JAD-170805

Lea, S. E. G., Wills, A. J., Leaver, L. A., Ryan, C. M. E., Bryant, C. M. L., \& Millar, L. (2009). A comparative analysis of the categorization of multidimensional stimuli: II. Strategic information search in humans (Homo sapiens) but not in pigeons (Columba livia). Journal of Comparative Psychology, 123(4), 406-420. https://doi.org/10.1037/a0016851

Lee, S. A. (2017). The boundary-based view of spatial cognition: A synthesis. Current Opinion in Behavioral Sciences, 16, 58-65. https://doi.org/10.1016/j.cobeha.2017.03.006

Lee, S. A., \& Spelke, E. S. (2010). Two systems of spatial representation underlying navigation. Experimental Brain Research, 206(2), 179188. https://doi.org/10.3390/brainsci7020017

Lee, S. A., Tucci, V., Sovrano, V. A., \& Vallortigara, G. (2015). Working memory and reference memory tests of spatial navigation in mice (Mus musculus). Journal of Comparative Psychology, 129(2), 189197. https://doi.org/10.1037/a0039129

Leonard, K., Tian, N., Ivanco, T. L., \& Kelly, D. M. (2018). Experience with featural-cue reliability influences featural- and geometric-cue use by mice (Mus musculus). Journal of Comparative Psychology, 132(1), 106. https://doi.org/10.1037/com0000096

Lithfous, S., Dufour, A., \& Despres, O. (2013). Spatial navigation in normal aging and the prodromal stage of Alzheimer's disease: Insights from imaging and behavioral studies. Ageing Research Reviews, 12, 201-203. https://doi.org/10.1016/j.arr.2012.04.007

Maes, E., De Filippo, G., Inkster, A. B., Lea, S. E. G., De Houwer, J., D’Hooge, R., ... Wills, A. J. (2015). Feature- versus rule-based generalization in rats, pigeons and humans. Animal Cognition, 18, 1267-1284. https://doi.org/10.1007/s10071-015-0895-8

Moffat, S. D. (2009). Aging and spatial navigation: What do we know and where do we go? Neuropsychology Review, 19, 478-489. https://doi.org/10.1007/s11065-009-9120-3

Moffat, S. D., \& Resnick, S. M. (2002). Effects of age on virtual environment place navigation and allocentric cognitive mapping. Behavioral Neuroscience, 116(5), 851-859. https://doi.org/10. 1037//0735-7044.116.5.851

Monacelli, A. M., Cushman, L. A., Kavcic, V., \& Duffy, C. J. (2003). Spatial disorientation in Alzheimer's disease: The remembrance of things passed. Neurology, 61(11), 1491-1497. https://doi.org/10. 1212/wnl.61.11.1491

O'Connor, D. H., Huber, D., \& Svoboda, K. (2009). Reverse engineering the mouse brain. Nature, 461, 923-929. https://doi.org/10.1038/nature08539

Oddo, S., Caccamo, A., Shepherd, J. D., Murphy, M. P., Golde, T. E., Kayed, R., ... LaFerla, F. M. (2003). Triple-transgenic model of Alzheimer's disease with plaques and tangles: Intracellular $A \beta$ and synaptic dysfunction. Neuron, 39(3), 409-421. https://doi.org/ 10.1016/s0896-6273(03)00434-3

Pearce, J. M. (1987) A model for stimulus generalization in Pavlovian conditioning. Psychological Review, 94(1), 61-73.

Pecchia, T., \& Vallortigara, G. (2010). View-based strategy for reorientation by geometry. Journal of Experimental Biology, 213, 29872996. https://doi.org/10.1242/jeb.043315

Price, D. L., Sisodia, S. S., \& Borchelt, D. R. (1998). Genetic neurodegenerative diseases: The human illness and transgenic models. Science, 282(5391), 1079-1083. https://doi.org/10.1126/science. 282.5391 .1079
Sasaguri, H., Nilsson, P., Hashimoto, S., Nagata, K., Saito, T., De Strooper, B., ... Saido, T. C. (2017). APP mouse models for Alzheimer's disease preclinical studies. EMBO Journal, 37(17), 2473-2487. https://doi.org/10.15252/embj.201797397

Serino, S., Cipresso, P., Morganit, F., \& Riva, G. (2014). The role of egocentric and allocentric abilities in Alzheimer's disease: A systematic review. Ageing Research Reviews, 16, 32-44. https://doi. org/10.1016/j.arr.2014.04.004

Siemens, M., \& Kelly, D. M. (2017, June). The effect of age and sex on spatial reorientation. Paper presentation at the Canadian Society for Brain, Behaviour and Cognitive Science, Saskatchewan, Canada.

Spetch, M. L., Cheng, K., \& MacDonald, S. E. (1996). Learning the configuration of a landmark array: I. Touch-screen studies with pigeons and humans. Journal of Comparative Psychology, 110(1), 55-68. https://doi.org/10.1037/0735-7036.110.1.55

Spetch, M. L., Cheng, K., MacDonald, S. E., Linkenhoker, B. A., Kelly, D. M., \& Doerkson, S. R. (1997). Use of landmark configuration in pigeons and humans: II. Generality across search tasks. Journal of Comparative Psychology, 111(1), 14-24. https://doi.org/10.1037/ 0735-7036.111.1.14

Sterniczuk, R., Antle, M. C., LaFerla, F. M., \& Dyck, R. H. (2010). Characterization of the 3xTg-AD mouse model of Alzheimer's disease: Part 2. Behavioral and cognitive changes. Brain Research, 1348, 149-155. https://doi.org/10.1016/j.brainres.2010.06.011

Stevens, L. M., \& Brown, R. E. (2015). Reference and working memory deficits in the 3xTg-AD mouse between 2 and 15 months of age: A cross-sectional study. Behavioural Brain Research, 278, 496-505. https://doi.org/10.1016/j.bbr.2014.10.033

Stimmell, A. C., Baglietto-Vargas, D., Moseley, S. C., Lapointe, V., Thompson, L. M., LaFerla, F. M., ... Wilber, A. A. (2019). Impaired spatial reorientation in the $3 \times \mathrm{Tg}-\mathrm{AD}$ mouse model of Alzheimer's disease. Scientific Reports, 9(1311). https://doi.org/10. 1038/s41598-018-37151-z

Stover, K. R., Campbell, M. A., Van Winssen, C. M., \& Brown, R. E. (2015). Early detection of cognitive deficits in the 3xTg-AD mouse model of Alzheimer's disease. Behavioural Brain Research, 289, 29-38. https://doi.org/10.1016/j.bbr.2015.04.012

Twyman, A. D., Newcombe, N. S., \& Gould, T. J. (2009). Of mice (Mus musculus) and toddlers (Homo sapiens): Evidence for speciesgeneral spatial reorientation. Journal of Comparative Psychology, 123, 342-345. https://doi.org/10.1037/a0015400

Twyman, A. D., Newcombe, N. S., \& Gould, T. J. (2013). Malleability in the development of spatial reorientation. Developmental Psychobiology, 55, 243-255. https://doi.org/10.1002/dev.21017

Vallortigara, G., Zanforlin, M., \& Pasti, G. (1990). Geometric modules in animals' spatial representations: A test with chicks (Gallus gallus domesticus). Journal of Comparative Psychology, 104(3), 248-254. https://doi.org/10.1037/0735-7036.104.3.248

Wills, A. J., Lea, S. E. G., Leaver, L. A., Osthaus, B., Ryan, C. M. E., Suret, M. B., ... Millar, L. (2009). A comparative analysis of the categorization of multidimensional stimuli: I. Unidimensional classification does not necessarily imply analytic processing; Evidence from pigeons (Columba livia), squirrels (Sciurus carolinensis), and humans (Homo sapiens). Journal of Comparative Psychology, 123(4), 391-405. https://doi.org/10.1037/a0016216

Wolbers, T., \& Hegarty, M. (2010). What determines our navigational abilities? Trends in Cognitive Sciences, 14(3), 138-146. https://doi. org/10.1016/j.tics.2010.01.001

Wystrach, A., Cheng, K., Sosa, S., \& Beugnon, G. (2011). Geometry, features, and panoramic views: Ants in rectangular arenas. Journal of Experimental Psychology: Animal Behavior Processes, 37(4), 420-435. https://doi.org/10.1037/a0023886

Publisher's note Springer Nature remains neutral with regard to jurisdictional claims in published maps and institutional affiliations. 